\title{
Associations between early-life screen viewing and 24 hour movement behaviours : findings from a longitudinal birth cohort study
}

\section{Chen, Bozhi}

2020-03

Chen , B , Bernard , J Y , Padmapriya , N , Ning , Y , Cai , S, Lanca , C , Tan , K H, Yap , F , Chong , Y-S , Shek , L, Godfrey, K M , Saw , S M , Chan , S-Y, Eriksson, J G , Tan , C S \& Muller-Riemenschneider , F 2020 , ' Associations between early-life screen viewing and 24 hour movement behaviours : findings from a longitudinal birth cohort study ' , Lancet child \& adolescent health , vol. 4 , no. 3 , pp. 201-209 . https://doi.org/10.1016/S2352-4642(19)30424-9

http://hdl.handle.net/10138/328539

https://doi.org/10.1016/S2352-4642(19)30424-9

draft

Downloaded from Helda, University of Helsinki institutional repository.

This is an electronic reprint of the original article.

This reprint may differ from the original in pagination and typographic detail.

Please cite the original version. 


\section{Associations between early-life screen viewing and 24 hour movement behaviours: findings from a longitudinal birth cohort study}

Bozhi Chen, Jonathan Y Bernard, Natarajan Padmapriya, Yilin Ning, Shirong Cai, Carla Lança, Kok Hian Tan, Fabian Yap, Yap-Seng Chong,

Lynette Shek, Keith M Godfrey, Seang Mei Saw, Shiao-Yng Chan, Johan G Eriksson, Chuen Seng Tan, Falk Müller-Riemenschneider

\section{Summary}

Background Screen viewing is a sedentary behaviour reported to interfere with sleep and physical activity. However, few longitudinal studies have assessed such associations in children of preschool age (0-6 years) and none have accounted for the compositional nature of these behaviours. We aimed to investigate the associations between total and device-specific screen viewing time at age 2-3 years and accelerometer-measured $24 \mathrm{~h}$ movement behaviours, including sleep, sedentary behaviour, light physical activity, and moderate-to-vigorous physical activity (MVPA) at age $5 \cdot 5$ years.

Methods The Growing Up in Singapore Towards healthy Outcomes (GUSTO) study is an ongoing longitudinal birth cohort study in Singapore, which began in June 2009. We recruited pregnant women during their first ultrasound scan visit at two major public maternity units in Singapore. At clinic visits done at age 2-3 years, we collected parentreported information about children's daily total and device-specific screen viewing time (television, handheld devices, and computers). At $5 \cdot 5$ years, children's movement behaviours for 7 consecutive days were measured using wristworn accelerometers. We assessed the associations between screen viewing time and movement behaviours (sedentary behaviour, light physical activity, MVPA, and sleep) using Dirichlet regression, which accounts for the compositional nature of such behaviours. This study is active but not recruiting and is registered with ClinicalTrials.gov, NCT01174875.

Findings Between June 1, 2009, and Oct 12, 2010, 1247 pregnant women enrolled and 1171 singleton births were enrolled. 987 children had parent-reported screen data at either 2 or 3 years, of whom 840 attended the clinic visit at age $5 \cdot 5$ years, and 577 wore an accelerometer. 552 children had at least 3 days of accelerometer data and were included in the analysis. Total screen viewing time at age $2-3$ years had a significant negative association with sleep $(p=0 \cdot 008)$, light physical activity $(\mathrm{p}<0 \cdot 0001)$, and MVPA $(\mathrm{p}<0.0001)$ in relation to sedentary behaviour at age $5 \cdot 5$ years. Compared with children who spent $1 \mathrm{~h}$ or less per day screen viewing at age 2-3 years, children who screen viewed for $3 \mathrm{~h}$ or more per day at 2-3 years engaged in more sedentary behaviour $(439.8 \mathrm{mins}$ per day [ $\leq 1 \mathrm{~h}$ screen viewing time] vs 480.0 mins per day [ $\geq 3 \mathrm{~h}$ screen viewing time]), and less light physical activity ( $384.6 \mathrm{vs} 356 \cdot 2 \mathrm{mins}$ per day), and MVPA (76.2 vs 63.4 mins per day) at age 5.5 years. No significant differences in time spent sleeping were observed between the groups $(539.5$ vs $540 \cdot 4$ mins per day). Similar trends were observed for television viewing and handheld device viewing.

Interpretation Longer screen viewing time in children aged 2-3 years was associated with more time spent engaged in sedentary behaviour and shorter time engaged in light physical activity and MVPA in later childhood. Our findings indicate that screen viewing might displace physical activity during early childhood, and suggest that reducing screen viewing time in early childhood might promote healthier behaviours and associated outcomes later in life.

Funding Singapore National Research Foundation, and Singapore Institute for Clinical Sciences, Agency for Science Technology and Research (A*STAR).

Copyright (C) 2020 Elsevier Ltd. All rights reserved.

\section{Introduction}

Screen viewing is increasingly prevalent and has become a part of common sedentary behaviour globally. Excessive screen viewing time has been reported to be associated with poorer health outcomes in children, including increased obesity risk, reduced motor and cognitive development, and worse psychosocial health. ${ }^{1}$ Considering the detrimental effects of excessive sedentary behaviour on health, $\mathrm{WHO}^{2}$ and several countries have developed and issued recommendations to limit screen time to $1 \mathrm{~h}$ per day or less among children aged 2-5 years. However, concerns about the recommendation for a universal cutoff for children's overall screen time have been raised, ${ }^{3,4}$ considering the relatively weak evidence and the differential effects of different types of screen time.
Lancet Child Adolesc Health 2020 Published Online January 28,2020 https://doi.org/10.1016/ S2352-4642(19)30424-9 See Online/Comment https://doi.org/10.1016/ S2352-4642(20)30005-5

Saw Swee Hock School of Public Health (B Chen MPH, N Padmapriya MSc,

Prof S M Saw PhD, C S Tan PhD, F Müller-RiemenschneiderMD), Departments of Obstetrics and Gynaecology (N Padmapriya, S Cai, ProfY-S Chong, Prof L Shek FRCOG, S-Y Chan, ProfJ G Eriksson), Surgery ( $\mathrm{Y}$ Ning $\mathrm{Bs} C$ ) and Paediatrics (Prof L Shek), Yong Loo Lin School of Medicine, and National University of Singapore Graduate School of Integrative Science and Engineering ( $Y$ Ning), National University of Singapore, Singapore; Centre for Research in Epidemiology and Statistics (CRESS), Université de Paris, National Institute of Health and Medical Research, Paris, France (JY BernardDrPH); Singapore Institute for Clinical Sciences, Agency for Science Technology and Research (A*STAR), Singapore (JY Bernard, S Cai PhD, Prof Y-S Chong MD, S-Y Chan PhD Prof J G Eriksson DMSc); Singapore Eye Research Institute, Singapore (C Lança PhD); Departments of Maternal Fetal Medicine (Prof K HTan FRCOG) and Paediatrics (FYap FRCOG), KK Women's and Children's Hospital, Singapore; Duke-NUS Medical School, Singapore (Prof K H Tan, FYap); Lee Kong Chian School of Medicine, Nanyang Technological University, Singapore (FYap); Division of Paediatric Allergy, Immunology and Rheumatology, Khoo Teck Puat-National University 
Children's Medical Institute, National University Health System, Singapore (Prof L Shek); Medical Research Council Lifecourse Epidemiology Unit, University of Southampton, Southampton, UK (Prof K M Godfrey PhD); NIHR Southampton Biomedical Research Centre, University of Southampton and University Hospital Southampton NHS Foundation Trust, Southampton, UK

(Prof K M Godfrey); Department of General Practice and Primary Health Care, University of Helsinki, Helsinki, Finland (ProfJ G Eriksson); Folkhalsan Research Center, Helsinki, Finland (Prof J G Eriksson); and Berlin Institute of Health, Charite University Medical Centre, Berlin, Germany (F Müller-Riemenschneider)

Correspondence to: Dr Falk Müller-Riemenschneider Saw Swee Hock School of Public Health, National University of Singapore, Singapore 117549 ephmf@nus.edu.sg

\section{Research in context}

\section{Evidence before this study}

Early childhood is a crucial period for developing behavioural habits that persist into adolescence and adulthood. Screen viewing has been suggested to have detrimental effects on child health, including an increased risk for obesity, reduced motor and cognitive development, and poorer psychosocial health. According to an estimation from UNICEF, WHO, and the World Bank, in 2016, more than 41 million children aged younger than 5 years were overweight and obese; the number has continued to increase during the past few years and is estimated to reach more than 50 million by 2030 . Possible mechanisms linking screen viewing time with obesity include the inverse associations between screen viewing time and physical activity and sleep. However, the longitudinal effects of screen viewing on movement behaviours within a finite $24 \mathrm{~h}$ period are unknown. To identify studies that have assessed the associations between screen viewing time and physical activity, sedentary behaviour, and sleep in children, we searched PubMed from database inception to Sept 17, 2019, for studies published in English using the primary search terms "children", "screen viewing", "media use", "television viewing", "physical activity", "exercise", "fitness", "sleep" and "movement behaviour". Most studies assessing associations between screen viewing time and physical activity and sleep included schoolaged children (age 6-12 years) and adolescents (age 12-19 years). A systematic review concluded that associations between screen time and physical activity were weak and inconsistent in school-aged children and adolescents. The literature consistently supports the importance of reducing screen viewing time for adequate sleep duration among children and adolescents. We further observed that most of the existing studies reporting on the association between screen viewing time and movement behaviours used a cross-sectional design. No previous study has assessed the associations between screen viewing time and movement behaviours using composition techniques, which take into account the compositional nature of movement behaviours within a defined $24 \mathrm{~h}$ period.

\section{Added value of this study}

By using analyses that account for compositional time use, we found that longer total and device-specific screen viewing time at age 2-3 years was associated with a higher proportion of time spent on sedentary behaviours and a lower level of moderateto-vigorous physical activity and light physical activity at age $5 \cdot 5$ years. These findings extend existing research in children of preschool and school age (age 0-12 years) through the use of a longitudinal study design and the objective assessments of all movement behaviours, thereby strengthening the existing evidence linking screen viewing time with later child health.

\section{Implications of all the available evidence}

Our analysis addresses an important research gap and establishes a platform for research to further assess the effect of content type, timing, and parental co-use of media with their children on unfavourable movement behaviours. The findings suggest that further research on the longitudinal effects of screen viewing on other movement behaviours is warranted, and support public health efforts to reduce screen viewing time in young children to minimise the possible negative impact on children's health behaviours and general health.
One of the suggested mechanisms through which screen viewing might influence health is by displacing time that would otherwise be spent engaged in physical activity. This proposed hypothesis has, to our knowledge, rarely been investigated in children of preschool age (age 0-6 years), but has been supported by evidence from studies in adolescents. On one hand, some studies have suggested that long periods of screen viewing are strongly associated with decreases in physical activity., ${ }^{5,6}$ On the other hand, a previous meta-analysis and a 2013 study reported that such associations are either weak or nonexistent. ${ }^{78}$ As such, evidence on the association between screen viewing time and physical activity is mixed and inconclusive. Inadequate sleep is another possible mechanism linking screen viewing time and poor health. School-aged children (age 6-12 years) and adolescents who spend excessive time watching screens might be more likely to have inadequate sleep, which is usually measured by a lower total sleep time and later bedtimes. ${ }^{9}$ Such associations are concerning since they develop during infancy and can persist in mid-childhood. ${ }^{10}$

However, studies investigating the associations between screen viewing time and other behaviours, such as physical activity and sleep, have mainly been done in school-aged children and adolescents (age 12-19 years), and adequate empirical evidence is scarce in young children. Moreover, most previous studies have been largely cross-sectional and thus findings could be affected by reverse causation. Previous studies have focused on conventional electronic devices such as televisions and computers, and little evidence is available regarding newer portable devices such as smartphones and tablets that have broad capabilities (eg, internet). Newer types of screen devices, which allow real-time interaction and potentially continuous stimulation for children, provide a different type of exposure and have become increasingly common in young children. ${ }^{11}$ Studies have also been criticised for investigating physical activity, sedentary behaviour, and sleep in isolation, or with only partial adjustment for time spent on other behaviours. ${ }^{12,13}$ This approach does not account for the fact that an increase in one behaviour might lead to a decrease in at least one of the remaining behaviours.

Physical activity, sedentary behaviour, and sleep represent the movement spectrum across $24 \mathrm{~h}$ and are 
referred to as movement behaviours. ${ }^{13}$ Since 2016, several individual countries and $\mathrm{WHO}^{2}$ have developed integrated movement guidelines for children, which highlight the importance of targeting all movement behaviours to maximize health benefits. Previous evidence from our GUSTO mother-offspring cohort in Singapore showed that the majority of children aged $5 \cdot 5$ years engaged in excessive screen viewing time, had inadequate sleep, and low levels of physical activity; only one in 20 children met all the integrated guideline targets. ${ }^{14}$ Such a high prevalence of unfavourable behaviours highlights the necessity of identifying opportunities to improve all these behaviours in young children. Previous studies have repeatedly suggested the conceptualisation of individuals' daily activity data as compositions, consisting of time spent asleep, sedentary behaviour, and different intensities of physical activity (ie, light physical activity and moderate-tovigorous physical activity [MVPA]), which sum to $24 \mathrm{~h} .{ }^{13,15} 24 \mathrm{~h}$ movement data require different analytical methods than traditional regressions, to account for their compositional nature. Although composition techniques have been proposed to be valid approaches for analysing a set of variables that sum to a constant ${ }^{16}$ and implemented in a number of research areas, ${ }^{17,18}$ few studies have implemented these techniques in movement behaviour research, ${ }^{15,19}$ and no studies have explored how screen viewing behaviour affects these activity components.

To address existing research gaps, we aimed to investigate the associations of total and device-specific screen viewing time at ages $2-3$ years with accelerometermeasured movement components, including sleep, sedentary behaviour, light physical activity, and MVPA at age $5 \cdot 5$ years.

\section{Methods \\ Study design}

Growing Up in Singapore Towards healthy Outcomes (GUSTO) is an ongoing longitudinal birth cohort study in Singapore, which began in June 2009. The study design and protocols have been reported elsewhere..$^{20}$ Briefly, pregnant women were recruited during their first ultrasound scan visit at two major public maternity units in Singapore (KK Women's and Children's Hospital and National University Hospital). Pregnant women who were Singaporean citizens or permanent residents of Chinese, Malay, or Indian ethnicity with a homogenous ethnic background, who intended to deliver in KK Women's and Children's Hospital or National University Hospital, and who intended to stay in Singapore for at least the next 5 years were eligible. All participants provided written informed consent for themselves and on behalf of their offspring. This study obtained ethical approval from the National Healthcare Group Domain Specific Review Board and the SingHealth Centralised Institutional Review Board.

\section{Procedures}

During clinic visits at age $2-3$ years, time spent on screen devices was assessed as part of a questionnaire about the child's outdoor and indoor activities. Trained interviewers asked the mother how much time (in 5-min increments), their child spent on average both on weekdays and weekends using the following screen devices: televisions (viewing or playing television games); computers; and handheld devices (eg, video games and hand phones including tablets). The activities done while using handheld devices were not specified. Mean daily devicespecific screen viewing time $(\mathrm{h})$ was calculated as follows: (time spent viewing screens per weekday $\times 5+$ time spent viewing screens per weekend day $\times 2$ ) divided by 7 , to calculate the mean time spent using televisions, computers, and handheld device each day. Total screen viewing time was calculated as the sum of time spent using the three types of screen devices. Screen viewing time was further categorised into three groups $(\leq 1 \mathrm{~h}$ per day, $1-3 \mathrm{~h}$ per day, and $\geq 3 \mathrm{~h}$ per day) for the main analyses. The lower $1 \mathrm{~h}$ cutoff point was chosen on the basis of international recommendations for children aged $2-5$ years of no more than $1 \mathrm{~h}$ of screen viewing time per day; ${ }^{2}$ the upper $3 \mathrm{~h}$ cutoff point was chosen on the basis of the data collected from the questionnaire. Specifically, an upper $3 \mathrm{~h}$ cutoff point was used because around one third of this sample engaged in $3 \mathrm{~h}$ or more of screen viewing time per day.

At clinic visits at age $5 \cdot 5$ years, movement behaviours including physical activity, sedentary behaviour, and sleep were assessed objectively using triaxial accelerometers (ActiGraph wGT3X+-BT; ActiGraph Pensacola, FL, USA), which children wore $24 \mathrm{~h}$ per day for 7 days. At the clinic visit, clinical staff attached an accelerometer with a non-removable strap on each child's non-dominant wrist. Children were asked to wear the accelerometer for 7 days and nights.

Data processing has been described in detail elsewhere. ${ }^{14}$ Briefly, the raw data files were processed in R-package GGIR (version 1.6-0), ${ }^{21}$ which autocalibrates the raw triaxial accelerometer signals and converts them into vector magnitude units corrected for gravity, termed the Euclidean Norm Minus One (ENMO). ${ }^{21}$ Files were excluded from all analyses if they failed to record a minimum of $16 \mathrm{~h}$ per day for at least 3 days of wearing time. Non-wear time was estimated on the basis of the SD and value range of each axis, using a 60 -min window with 15-min increments. ${ }^{22}$ Sleep duration (night time and daytime sleep) was estimated using the method described by van Hees and colleagues as part of GGIR processing. ${ }^{23}$ According to Hildebrand and colleagues' prediction equations, ${ }^{24,25}$ the following acceleration intensity thresholds were used to classify activity during the waking period: sedentary behaviour (ENMO $\leq 35$ milligravitational units $[\mathrm{mg}]$ ), light physical activity (ENMO 35-200 mg), and MVPA (ENMO >200 mg). Since each included participant had at least 3 valid days of data, 


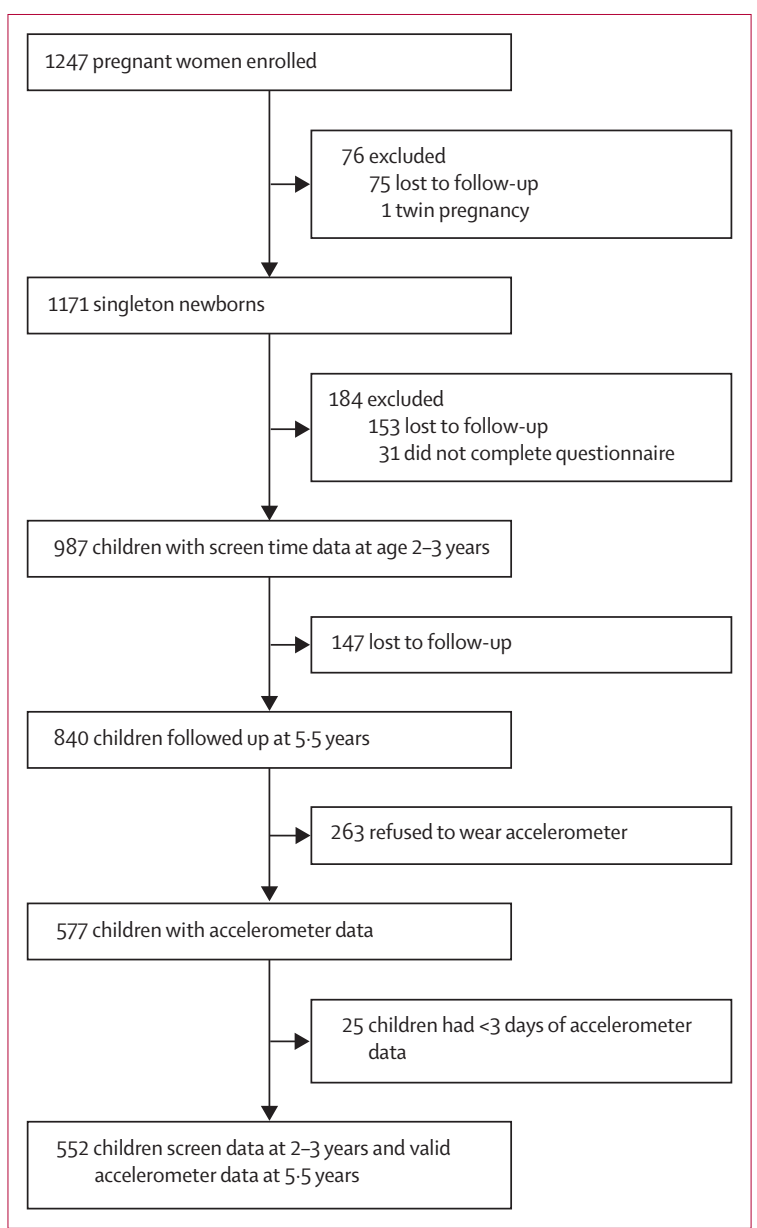

Figure: Study flow diagram

weekdays were weighted so that all weekdays together had a weight of five-sevenths, and weekend days were similarly weighted so that all weekend days had a weight of two-sevenths, making the data representative of a full week.

\section{Covariates}

As part of interviewer-administered questionnaires, sociodemographic information was obtained at enrolment, including ethnicity (Chinese, Malay, Indian; all children had homogenous parentage), highest level of maternal education (primary or secondary, postsecondary, or university), monthly household income $(<4000,4000-5999$, or $\geq 6000$ S\$). Maternal pre-pregnancy weight was self-reported; maternal height was measured at 26-28 weeks' gestation using a stadiometer (seca model 213; Seca, Hamburg, Germany). Children's height and weight were measured at the 2-year clinic visit, using the same stadiometer and a weighing scale (SECA model 803; Seca). These measurements were used to calculate maternal pre-pregnancy body-mass index (BMI) and child BMI. Information on maternal age at delivery and offspring's date of birth, sex, and birth order was extracted from medical records.

\section{Statistical analysis}

To assess whether children included in the present analyses were different from those who were not included in the analyses (ie, singleton newborns not included in the analyses), we used Fisher's exact test and Student's $t$ test to compare categorical and continuous sociodemographic characteristics.

We used the mean of both screen viewing time measures when available $(n=523)$ to improve the precision of the screen viewing time variable. When only a single screen viewing time measure was available at either 2 or 3 years of age, we used that measure instead of the mean viewing time to maximize our sample size. The mean proportion of time spent on each movement component per day (sleep, sedentary behaviour, light physical activity, MVPA) for the accelerometer wearing time were calculated and used in further regression analyses. We used Dirichlet regression to simultaneously model the proportions of all components at age 5.5 years, because the proportions of all movement components for each child, by definition, always sum to $1 .^{26}$ To assess the effects of screen viewing time on the relative distributions of different components of $24 \mathrm{~h}$ movement patterns, so-called alternative parameterization was used, as proposed by Maier. ${ }^{27}$ The parametrization for the expected proportion (ie, mean) is similar to multinomial regression: we used sedentary behaviour time as the reference category and logit function to link the mean with the systematic component, which is a linear combination of predictors. ${ }^{28}$ Thus, the parameter of each predictor can be interpreted as an odds ratio (OR) after being exponentiated, ${ }^{28}$ which eases interpretation of the associations. With regard to dispersion parameter, we first fitted two models (one with constant and one with varying dispersion) and then compared them using likelihood ratio tests. No significant difference in model fit was found between the two models ( $>00.05)$; therefore, we assumed a common dispersion parameter for all participants.

We used Dirichlet regression models to examine adjusted associations between screen viewing time (total, television, handheld devices) and the proportion of time spent engaged in each movement behaviour. The multivariable models consisted of sociodemographic variables, including the child's sex, ethnicity, birth order, BMI at age 2 years, household income, and maternal education level, pre-pregnancy BMI and age at delivery, and study centre. In models with television and handheld devices viewing times as exposures, each exposure was adjusted for the other device-specific viewing time. We developed additional regression models using continuous screen viewing time (h per day) at 2-3 years. In addition to providing OR, we obtained the marginal mean of the proportion for each component at $5 \cdot 5$ years and multiplied by 1440 to 


\begin{tabular}{|c|c|c|}
\hline & $\begin{array}{l}\text { Included } \\
\text { children } \\
(n=552)\end{array}$ & $\begin{array}{l}\text { Excluded } \\
\text { children } \\
(n=619)\end{array}$ \\
\hline Age (years) & $5 \cdot 5(0 \cdot 1)$ & $5 \cdot 5(0 \cdot 1)$ \\
\hline \multicolumn{3}{|l|}{ Sex } \\
\hline Boys & $287(52 \cdot 0 \%)$ & $332(53.6 \%)$ \\
\hline Girls & $265(48.0 \%)$ & $287(46 \cdot 4 \%)$ \\
\hline \multicolumn{3}{|l|}{ Ethnicity } \\
\hline Chinese & $323(58 \cdot 5 \%)$ & $337(54 \cdot 4 \%)$ \\
\hline Malay & $136(24 \cdot 6 \%)$ & $163(26 \cdot 3 \%)$ \\
\hline Indian & $93(16 \cdot 8 \%)$ & $119(19 \cdot 2 \%)$ \\
\hline \multicolumn{3}{|l|}{ Birth order } \\
\hline First-born & $242(43 \cdot 8 \%)$ & $293(47 \cdot 3 \%)$ \\
\hline Second- or later-born & $310(56 \cdot 2 \%)$ & $326(52 \cdot 7 \%)$ \\
\hline Child BMI at age 2 years $\left(\mathrm{kg} / \mathrm{m}^{2}\right)$ & $16 \cdot 2(1 \cdot 4)$ & $16 \cdot 2(1 \cdot 5)$ \\
\hline \multicolumn{3}{|l|}{ Monthly household income ( $\$ \$)$} \\
\hline$<4000$ & $238(43 \cdot 1 \%)$ & $263(42 \cdot 5 \%)$ \\
\hline 4000-5999 & $119(21 \cdot 6 \%)$ & $154(24 \cdot 9 \%)$ \\
\hline$\geq 6000$ & $161(29 \cdot 2 \%)$ & $159(25 \cdot 7 \%)$ \\
\hline missing, $n$ & $34(6 \cdot 2 \%)$ & $43(6 \cdot 9 \%)$ \\
\hline \multicolumn{3}{|l|}{ Maternal age at delivery (years) } \\
\hline$<30$ & $224(40 \cdot 6 \%)$ & $269(43 \cdot 5 \%)$ \\
\hline $30-35$ & $172(31 \cdot 2 \%)$ & $221(35 \cdot 7 \%)$ \\
\hline$\geq 35$ & $156(28 \cdot 3 \%)$ & $129(20 \cdot 8 \%)$ \\
\hline \multicolumn{3}{|l|}{ Maternal education } \\
\hline Primary or secondary & $172(31 \cdot 2 \%)$ & $179(28.9 \%)$ \\
\hline Post-secondary & $188(34 \cdot 1 \%)$ & $221(35 \cdot 7 \%)$ \\
\hline University & $188(34 \cdot 1 \%)$ & $206(33 \cdot 3 \%)$ \\
\hline Unknown & $4(0.7 \%)$ & $13(2 \cdot 1 \%)$ \\
\hline Maternal pre-pregnancy BMI $\left(\mathrm{kg} / \mathrm{m}^{2}\right)$ & $22 \cdot 6(4 \cdot 2)$ & $23 \cdot 0(4 \cdot 7)$ \\
\hline \multicolumn{3}{|l|}{ Study centre } \\
\hline $\begin{array}{l}\text { KK Women's and Children's } \\
\text { Hospital }\end{array}$ & $418(75 \cdot 7 \%)$ & $473(76 \cdot 4 \%)$ \\
\hline National University Hospital & $134(24 \cdot 3 \%)$ & $146(23 \cdot 6 \%)$ \\
\hline \multicolumn{3}{|c|}{$\begin{array}{l}\text { Data are mean (SD) or } n \text { (\%). Fisher's exact test for categorical variables and } \\
\text { Student's } t \text { test for continuous variables were used to compare children included } \\
\text { in the study with those not included (ie, } 619 \text { of } 1171 \text { singleton newborns born in } \\
\text { the study period, who were not included in the analyses). No significant } \\
\text { differences were identified between the groups for any characteristic ( } p>0.05 \text { for } \\
\text { all). BMl=body-mass index. } \$ \$=\text { Singapore dollars. }\end{array}$} \\
\hline
\end{tabular}

estimate its corresponding time in minutes per day (appendix p 1).

We did sensitivity analyses, in which we did additional Dirichlet regressions using complete screen viewing data at 2 years $(n=531), 3$ years $(n=541)$, and both 2 and 3 years $(\mathrm{n}=523)$. To reduce residual confounding, we further controlled for outdoor physical activity and non-screenbased sedentary behaviour at 2 years. In addition to Dirichlet regression, we also applied the commonly used compositional data analysis approach, ${ }^{16,29}$ to assess the robustness of our results.

All statistical analyses were done using $\mathrm{R}$ statistical software (version 3.4.3). The Dirichlet regression was

\begin{tabular}{|c|c|}
\hline & Children $(n=552)$ \\
\hline \multicolumn{2}{|l|}{ Total screen viewing time (h per day) } \\
\hline$\leq 1$ & $107(19 \cdot 4 \%)$ \\
\hline $1-3$ & $285(51 \cdot 6 \%)$ \\
\hline$\geq 3$ & $160(29 \cdot 0 \%)$ \\
\hline $\operatorname{Mean}(\mathrm{SD})^{*}$ & $2 \cdot 5(1 \cdot 8)$ \\
\hline \multicolumn{2}{|l|}{ Television ( $\mathrm{h}$ per day) } \\
\hline$\leq 1$ & $227(41 \cdot 1 \%)$ \\
\hline $1-3$ & $241(43 \cdot 7 \%)$ \\
\hline$\geq 3$ & $84(15 \cdot 2 \%)$ \\
\hline Mean $(S D)^{*}$ & $1 \cdot 6(1 \cdot 3)$ \\
\hline \multicolumn{2}{|l|}{ Handheld devices (h per day) } \\
\hline$\leq 1$ & $418(75 \cdot 7 \%)$ \\
\hline $1-3$ & $115(20 \cdot 8 \%)$ \\
\hline$\geq 3$ & $19(3 \cdot 4 \%)$ \\
\hline Mean $(S D)^{*}$ & $0.8(0.8)$ \\
\hline \multicolumn{2}{|l|}{ Computer (h per day) } \\
\hline$\leq 1$ & $541(98 \cdot 0 \%)$ \\
\hline $1-3$ & $11(2 \cdot 0 \%)$ \\
\hline$\geq 3$ & 0 \\
\hline Mean $(S D)^{*}$ & $0.1(0 \cdot 3)$ \\
\hline \multicolumn{2}{|c|}{ 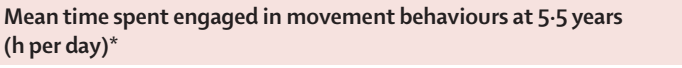 } \\
\hline Sleep & $9 \cdot 0(1 \cdot 0)$ \\
\hline Sedentary behaviour & $7 \cdot 7(1.1)$ \\
\hline Light physical activity & $6 \cdot 1(0.9)$ \\
\hline Moderate-to-vigorous physical activity & $1 \cdot 1(0 \cdot 4)$ \\
\hline \multicolumn{2}{|c|}{$\begin{array}{l}\text { Data are } n(\%) \text { or mean (SD). *Data presented as the mean number of h spent } \\
\text { engaged in each movement category; time spent wearing the accelerometer eact } \\
\text { day for each child was normalised to } 24 \mathrm{~h} \text {. }\end{array}$} \\
\hline \multicolumn{2}{|c|}{$\begin{array}{l}\text { Table 2: Parent-reported time spent engaged in screen viewing at age } \\
\text { 2-3 years, and accelerometer-measured physical activity, sedentary } \\
\text { behaviour, and sleep at age } 5.5 \text { years in the GUSTO cohort ( } n=552 \text { ) }\end{array}$} \\
\hline
\end{tabular}

done using the DirichletReg package. ${ }^{27}$ This study is active but not recruiting and is registered with ClinicalTrials. gov, NCT01174875.

\section{Role of the funding source}

The funders had no role in study design, data collection, data analysis, data interpretation, or writing of the report. The corresponding author had full access to all the data in the study and had final responsibility for the decision to submit for publication.

See Online for appendix

\section{Results}

Between June 1, 2009, and Oct 12, 2010, 1247 pregnant women enrolled and 1171 singleton births were enrolled. Of the 987 children who had parent-reported screen data at either 2 or 3 years, 840 attended the clinic visit at age $5 \cdot 5$ years, and 577 wore an accelerometer. Of the 577 children who wore an accelerometer, 552 had at least 3 days of accelerometer data and thus were included in the analysis (figure). Children included in the present analysis (552 of 1171 singleton births) 


\begin{tabular}{|c|c|c|c|c|c|c|}
\hline & \multicolumn{2}{|l|}{ Sleep } & \multicolumn{2}{|c|}{ Light physical activity } & \multicolumn{2}{|c|}{$\begin{array}{l}\text { Moderate-to-vigorous } \\
\text { physical activity }\end{array}$} \\
\hline & OR $(95 \% \mathrm{Cl})$ & pvaluet & OR (95\% Cl) & p valuet & OR $(95 \% \mathrm{Cl})$ & p value \\
\hline \multicolumn{7}{|c|}{ Total screen viewing time ( $\mathrm{h}$ per day) } \\
\hline$\leq 1 \mathrm{~h}$ & 1 (ref) & 0.0081 & .. & $<0.0001$ & .. & $<0.0001$ \\
\hline $1-3$ & $0.97(0.92-1.02)$ &.. & $0.90(0.85-0.95)$ & .. & $0.86(0.78-0.95)$ & .. \\
\hline$\geq 3$ & $0.92(0.87-0.97)$ & .. & $0.85(0.80-0.90)$ &. & $0.76(0.68-0.85)$ & .. \\
\hline \multicolumn{7}{|c|}{ Television ( $\mathrm{h}$ per day) } \\
\hline$\leq 1 \mathrm{~h}$ & 1 (ref) & 0.2605 & .. & 0.0003 & .. & 0.0016 \\
\hline $1-3$ & $0.97(0.93-1.01)$ & .. & $0.92(0.88-0.96)$ & .. & $0.88(0.81-0.96)$ & .. \\
\hline$\geq 3$ & $0.98(0.92-1.04)$ &. & $0.91(0.85-0.97)$ & .. & $0.84(0.74-0.95)$ & .. \\
\hline \multicolumn{7}{|c|}{ Handheld devices (h per day) } \\
\hline$\leq 1 \mathrm{~h}$ & 1 (ref) & 0.4615 & .. & 0.0305 & .. & 0.0316 \\
\hline 1-3 h/day & $0.98(0.93-1.03)$ & .. & $0.93(0.88-0.98)$ & .. & $0.88(0.80-0.97)$ & .. \\
\hline$\geq 3 \mathrm{~h} /$ day & $0.94(0.85-1.05)$ &. & $1.02(0.91-1.15)$ & .. & $1.01(0 \cdot 82-1 \cdot 24)$ &. \\
\hline
\end{tabular}

$\mathrm{OR}=$ odds ratio. $\mathrm{BMI}=$ body-mass index. *Dirichlet regression model adjusted for child's sex, ethnicity, birth order, BMI at age 2 years, household income, and maternal education level, pre-pregnancy BMI, age at delivery, and study centre; in models with television and handheld devices viewing times as exposures, each exposure was adjusted for the other device-specific viewing time; sedentary behaviour was the reference activity component and its regression coefficients were set to zero. WWald test was used to assess overall $p$ value, indicating any differences in the proportion of time spent on physical activity or sleeping across the three categories of screen viewing time.

Table 3: Adjusted associations* between total and device-specific categorical screen viewing time ( $\leq 1$, $1-3, \geq 3 \mathrm{~h}$ per day) at age 2-3 years and the proportion of time spent engaged in activity components at age 5.5 years $(n=552)$

\begin{tabular}{|c|c|c|c|c|c|c|}
\hline & \multicolumn{2}{|l|}{ Sleep } & \multicolumn{2}{|c|}{ Light physical activity } & \multicolumn{2}{|c|}{$\begin{array}{l}\text { Moderate-to-vigorous } \\
\text { physical activity }\end{array}$} \\
\hline & OR $(95 \% \mathrm{Cl})$ & p value & OR $(95 \% \mathrm{Cl})$ & pvalue & $\mathrm{OR}(95 \% \mathrm{Cl})$ & $p$ value \\
\hline $\begin{array}{l}\text { Total screen viewing } \\
\text { time (h per day) }\end{array}$ & $\begin{array}{l}0.99 \\
(0.97-1.00)\end{array}$ & 0.0105 & $\begin{array}{l}0.97 \\
(0.96-0.99)\end{array}$ & $<0.0001$ & $\begin{array}{l}0.95 \\
(0.93-0.97)\end{array}$ & $<0.0001$ \\
\hline $\begin{array}{l}\text { Television } \\
\text { (h per day) }\end{array}$ & $\begin{array}{l}0.99 \\
(0.98-1.01)\end{array}$ & 0.3343 & $\begin{array}{l}0.98 \\
(0.96-0.99)\end{array}$ & 0.0111 & $\begin{array}{l}0.96 \\
(0.93-0.99)\end{array}$ & 0.0103 \\
\hline $\begin{array}{l}\text { Handheld devices } \\
\text { (h per day) }\end{array}$ & $\begin{array}{l}0.98 \\
(0.96-1.00)\end{array}$ & 0.0999 & $\begin{array}{l}0.97 \\
(0.95-1.00)\end{array}$ & 0.0491 & $\begin{array}{l}0.94 \\
(0.90-0.99)\end{array}$ & 0.0278 \\
\hline
\end{tabular}

$\mathrm{OR}=$ odds ratio. $\mathrm{BMI}=$ body-mass index. *Dirichlet regression model adjusted for child's sex, ethnicity, birth order, BMI at age 2 years, household income, maternal education level, pre-pregnancy BMI, maternal age at delivery, and study centre; in models with television and handheld devices viewing times as exposures, each exposure was adjusted for the other device-specific viewing time; sedentary behaviour was the reference activity component and its regression coefficients were set to zero.

Table 4: Adjusted associations* between total and device-specific continuous screen viewing time (h per day) at age 2-3 years and the proportion of time spent engaged in activity components at age 5.5 years $(n=552)$
Total screen viewing time was significantly associated with sleep $(\mathrm{p}=0 \cdot 008)$, light physical activity $(\mathrm{p}<0 \cdot 0001)$, and MVPA ( $<<0 \cdot 0001 ;$ table 3$)$. Specifically, compared with children who engaged in $1 \mathrm{~h}$ of screen viewing time or less per day, children who engaged in $3 \mathrm{~h}$ or more of screen viewing time at $2-3$ years spent a lower proportion of time engaged in movement behaviours than sedentary behaviour at 5.5 years (adjusted OR $0.92[95 \% \mathrm{CI}$ $0 \cdot 87-0 \cdot 97]$ for sleep; $0 \cdot 85$ [0.80-0.90] for light physical activity; and $0.76[0 \cdot 68-0 \cdot 85]$ for MVPA). The association seemed to be strongest between screen viewing time and MVPA. Significant dose-response relationships were identified between total screen viewing time and sleep (OR 0.99 [95\% CI 0.97-1.00]), light physical activity (0.97 [0.96-0.99]), and MVPA (0.95 [0.93-0.97]; table 4).

Relative to sedentary behaviour, no association was identified between the proportion of time spent sleeping and device-specific screen viewing time for television and handheld devices, whereas the association between time spent in other movement behaviours relative to sedentary behaviour was statistically significant. In particular, time spent on television viewing was negatively associated with light physical activity and MVPA; similar negative associations also persisted for $1-3 \mathrm{~h}$ of viewing time on handheld devices. Screen viewing time for television and handheld devices was more strongly associated with MVPA than light physical activity (table 3 ). In analyses using continuous screen viewing variables, our findings were similar: significant dose-response relationships were found between device-specific screen viewing time and light physical activity and MVPA, but not sleep (table 4). In sensitivity analyses, we observed similar results (appendix p 2).

Compared with children who spent $3 \mathrm{~h}$ or more per day screen watching at age $2-3$ years, children who spent $1 \mathrm{~h}$ or less per day screen watching were sedentary for a shorter time $(439.8$ mins per day $[\leq 1 \mathrm{~h}$ screen viewing time] vs 480.0 mins per day [ $\geq 3 \mathrm{~h}$ screen viewing time]) and spent more time doing light physical activity (384.6 vs 356.2 mins per day) and MVPA (76.2 vs 63.4 mins per day) at age $5 \cdot 5$ years; however, no substantial differences in sleep duration were observed between the groups (539.5 vs 540.4 mins per day; table 5). Similar trends were observed for television viewing and handheld device viewing time (table 5).

were similar to those not included (619 of 1171 singleton births) with regard to sociodemographic variables (table 1).

At age $2-3$ years, children spent a mean of $2 \cdot 5 \mathrm{~h}$ per day (SD 1.8) watching screen devices, mainly television and handheld devices (table 2). Considering that computers were used by a small minority of children and that children spent relatively little time using computers, data on computer screen viewing time was not assessed separately in subsequent analyses. With regard to movement behaviours assessed by accelerometry at age $5 \cdot 5$ years, children wore the device for a mean of $6 \cdot 9$ days (SD 1.5) for a mean of $23.8 \mathrm{~h}$ per day (SD 0.6).

\section{Discussion}

We found that children who spent a longer time viewing screens at age 2-3 years were less likely to spend time sleeping and to engage in light physical activity and MVPA in relation to sedentary behaviour at age $5 \cdot 5$ years compared with children who spent less time viewing screens as toddlers. In absolute terms, children with more screen viewing time in toddlerhood spent more time engaged in sedentary behaviour and less time doing light physical activity and MVPA at older ages. Time 
spent sleeping at $5 \cdot 5$ years did not differ substantially with regard to screen viewing time among children at 2-3 years. Television viewing time across all three categories $(\leq 1 \mathrm{~h}, 1-3 \mathrm{~h}$, and $\geq 3 \mathrm{~h}$ ) and viewing times of less than $3 \mathrm{~h}$ using handheld devices at 2-3 years were negatively associated light physical activity and MVPA.

In our study, we accounted for the nature of compositional data to provide a comprehensive investigation of all movement behaviours. This approach extends the available evidence, because despite the importance of considering the proportional nature of $24 \mathrm{~h}$ movement data, to our knowledge no study to date has assessed the associations between screen viewing time and movement behaviours using compositional techniques. Previous studies have reported that screen viewing time is positively associated with adiposity in children, and proposed displacement hypothesis as one mechanism underlying the associations. ${ }^{30}$ According to this hypothesis, screen viewing time is assumed to reduce energy expenditure by displacing physical activity. This displacement hypothesis has received some support, mainly from studies in older children and adolescents. ${ }^{31}$ Existing evidence in preschool-aged children, although limited, also suggests an inverse association between screen viewing time and physical activity time: in a cross-sectional study, DuRant and colleagues reported that children aged 3-4 years who spent a longer time watching television engaged in less physical activity than those who spent less time watching television. ${ }^{32}$ Our study found that longer screen viewing time in early life was associated with a reduction in physical activity later in life, which might indicate time displacement from physical activity at $5 \cdot 5$ years. Our findings thereby provide strong evidence to support the displacement hypothesis. Additionally, our study in children aged 2-3 years extends the existing evidence considerably into early life, demonstrating consistent longitudinal associations between screen viewing time and physical activity of different intensities.

Another hypothesis is that media exposure might have indirect effects on health outcomes through sleep deprivation. Inadequate sleep has been reported to be associated with developmental problems such as increased risk of obesity and attention-deficit hyperactivity disorder in children of preschool and school age. ${ }^{33}$ Previous evidence also suggested negative associations between sleep and screen viewing time across various screen devices (eg, television and handheld device) in children of similar age. ${ }^{9,34}$ In the present study, however, sleep duration did not differ substantially with regard to screen viewing time among children aged 2-3 years. Inconsistencies with previous studies might be due to variations in study design and sleep measurements, but could also reflect the establishment of more consistent bedtime routines after children attend preschools. Understanding the characteristics of the screen utilization patterns that are most disruptive to sleep, such as usage

\begin{tabular}{|c|c|c|c|c|}
\hline & $\begin{array}{l}\text { Sedentary } \\
\text { behaviour } \\
\text { (mins per } \\
\text { day) }\end{array}$ & $\begin{array}{l}\text { Sleep (mins } \\
\text { per day) }\end{array}$ & $\begin{array}{l}\text { Light physical } \\
\text { activity (mins } \\
\text { per day) }\end{array}$ & $\begin{array}{l}\text { Moderate-to- } \\
\text { vigorous } \\
\text { physical } \\
\text { activity (mins } \\
\text { per day) }\end{array}$ \\
\hline \multicolumn{5}{|c|}{ Total screen viewing time (h per day) } \\
\hline$\leq 1$ & $439 \cdot 8$ & $539 \cdot 5$ & $384 \cdot 6$ & $76 \cdot 2$ \\
\hline $1-3$ & $461 \cdot 4$ & $547 \cdot 7$ & $362 \cdot 0$ & $68 \cdot 9$ \\
\hline$\geq 3$ & $480 \cdot 0$ & $540 \cdot 4$ & $356 \cdot 2$ & 63.4 \\
\hline \multicolumn{5}{|c|}{ Television ( $\mathrm{h}$ per day) } \\
\hline$\leq 1$ & $450 \cdot 8$ & $541 \cdot 6$ & $374 \cdot 8$ & $72 \cdot 8$ \\
\hline $1-3$ & $469 \cdot 7$ & $544 \cdot 7$ & $358 \cdot 8$ & $66 \cdot 8$ \\
\hline$\geq 3$ & $471 \cdot 4$ & $549 \cdot 4$ & $355 \cdot 7$ & 63.5 \\
\hline \multicolumn{5}{|c|}{ Handheld devices ( $h$ per day) } \\
\hline$\leq 1 \mathrm{~h}$ & $458 \cdot 4$ & $543 \cdot 5$ & $367 \cdot 8$ & $70 \cdot 3$ \\
\hline $1-3 h$ & $474 \cdot 5$ & $550 \cdot 0$ & $352 \cdot 5$ & $63 \cdot 1$ \\
\hline$\geq 3 \mathrm{~h}$ & $472 \cdot 2$ & $526 \cdot 3$ & 373.6 & $67 \cdot 9$ \\
\hline \multicolumn{5}{|c|}{$\begin{array}{l}\text { BMI=body-mass index. *Estimated marginal means of daily mins spent in each } \\
\text { movement behaviour from the Dirichlet regression model that included the } \\
\text { screen viewing time and the following variables in the model: child's sex, } \\
\text { ethnicity, birth order, BMI at age } 2 \text { years, household income, and maternal } \\
\text { education level, pre-pregnancy BMI, age at delivery, and study centre; in models } \\
\text { with television and handheld device viewing times as exposures, each exposure } \\
\text { was adjusted for the other device-specific viewing time. }\end{array}$} \\
\hline \multicolumn{5}{|c|}{$\begin{array}{l}\text { Table 5: Estimated time spent engaged in movement behaviours* at } \\
5.5 \text { years by screen viewing time }\end{array}$} \\
\hline
\end{tabular}

before sleep, content of the programming, or interactivity of the user, would be useful. For example, research has indicated that psychological stimulation from media content (eg, violent or frightening media before bedtime) could mediate the effect of screen media use on sleep..$^{35}$ Further research to comprehensively investigate associations between screen viewing time and sleep in preschool-aged children, rather than only focusing on sleep duration, is warranted to better understand these associations.

In the present study, children spent on average more than $2 \mathrm{~h}$ per day watching screen devices at 2-3 years, and only a small proportion of children met WHO recommendations of $1 \mathrm{~h}$ per day or less of screen viewing time. ${ }^{2}$ Television was the most commonly used screen device and was associated with the longest viewing time. Additionally, children spent more than 30 mins per day using handheld devices. These results were consistent with previous findings ${ }^{14,30}$ from this cohort and other existing evidence among children aged 3 years or younger. ${ }^{34,36}$ In the present study, no clear threshold effects were observed across screen viewing time categories and we instead found a dose-response relationship whereby estimated daily time spent engaging in light physical activity and MVPA decreased while sedentary behaviour time increased. These findings suggest that even small amounts of daily screen viewing could have negative effects on health behaviours, supporting recent evidence from the $\mathrm{UK}^{3}$ and France. ${ }^{4}$ According to previous research done in the GUSTO 
cohort, only $5 \cdot 5 \%$ of Singaporean children aged $5 \cdot 5$ years met integrated 24-Hour Movement Guidelines, which is comparable with other Asian countries. ${ }^{14}$ The low adherence to the integrated movement guidelines is concerning, because adhering to the recommendations for all movement behaviours is associated with the lowest risk of obesity and other non-communicable diseases when compared with adhering to only one or none of the individual guidelines. ${ }^{33}$ Strategies to promote movement-related behaviours comprehensively represent an important public health target. Our findings illustrate that such strategies should include elements to reduce screen viewing time in early life.

Strengths of this study include its longitudinal design, objective assessment of movement behaviours, and the use of $24 \mathrm{~h}$ accelerometer data rather than a combination of approaches to measure all behaviours. Additionally, the application of Dirichlet regression approach accounted for the compositional nature of movement data. We also assessed exposure to different types of screen devices to reflect the recent technological advances. However, our study also has several limitations. First, parent-reported screen viewing data might be susceptible to recall bias. However, screen viewing time was measured at ages 2 and 3 years and the mean was included in analysis. Thus, such error might have been reduced. Second, this study did not control for other baseline health behaviours (eg, diet, sleep, or physical activity at 2 years) or environmental factors (eg, time spent in childcare) because little relevant data were available, which might have resulted in residual confounding. To partly address this concern, we did additional sensitivity analyses that further included outdoor physical activity and non-screenbased sedentary behaviour at 2 years, and the findings were similar. Third, although the use of wrist-worn accelerometers in young children has advantages, ${ }^{37}$ particularly in the monitoring of movement behaviours across a $24 \mathrm{~h}$ period, we acknowledge that the raw acceleration cutoffs are not validated in our study population. However, such cutoffs have been validated in other populations and to the best of our knowledge, no other cutoffs have been developed for preschool-aged children. Fourth, Dirichlet regression might have limitations for the analysis of movement behaviour data because of the assumption that the compositions are independent except for the simplex constraint. However, presented results were similar when compared with those from the compositional data analysis approach in the sensitivity analysis. Fifth, the GUSTO cohort does not represent the entire Singaporean population. For example, Malay and Indian families were purposely overrepresented at inclusion to ensure an adequate sample size was obtained for each ethnic group included; recruited mothers were also less likely to hold a university degree than the women from the general population of the same age range. ${ }^{20}$ Generalising our results should therefore be done with caution.
Our findings suggest that a longer screen viewing time in children aged 2-3 years is associated with more sedentary behaviour and less engagement in MVPA and light physical activity in later childhood. Such findings demonstrate that the displacement of physical activity by screen-based sedentary behaviour could already occur at age 2-3 years. Considering the substantial amount of screen viewing time at ages 2-3 years and its negative impact on movement behaviours and health later in life, strategies to reduce screen viewing time during the early years are needed to address this important public health threat. Further research into the potentially longitudinal effects of screen viewing on movement behaviours is warranted, to confirm and extend our findings.

\section{Contributors}

KHT, FY, Y-SC, LS, KMG, and S-YC conceived and designed the cohort study. BC, JYB, JGE, CST, and FM-R designed this study. NP, SC, and SMS contributed to data collection. BC, JYB, NP, YN, CL, CST, and FM-R contributed to data analysis and interpretation. BC drafted the manuscript, and JYB, YN, SC, KMG, SMS, S-YC, JGE, CST, and FM-R contributed important intellectual content. All authors read and approved the final manuscript.

\section{Funding}

This research was supported by the Singapore National Research Foundation under its Translational and Clinical Research (TCR) Flagship Programme and administered by the Singapore Ministry of Health's National Medical Research Council (NMRC), Singapore-

NMRC/TCR/004-NUS/2008; NMRC/TCR/012-NUHS/2014. Additiona funding was provided by the Singapore Institute for Clinical Sciences, Agency for Science Technology and Research (A*STAR), Singapore. KMG is supported by the National Institute for Health Research through the NIHR Southampton Biomedical Research Centre and by the European Union's Erasmus+ Capacity-Building ENeA SEA Project and Seventh Framework Programme (FP7/2007-2013), projects

EarlyNutrition and ODIN under grant agreement numbers 289346 and 613977. All participating pregnant women signed written informed consent for themselves and on behalf of their offspring at enrolment. The study received ethical approval from the National Healthcare Group Domain Specific Review Board and the SingHealth Centralised Institutional Review Board.

\section{Declaration of interests}

KMG and Y-SC report reimbursement for speaking at conferences sponsored by Nestle Nutrition Institute and are part of an academic consortium that has received research funding from Abbott Nutrition, Nestle, and Danone. Y-SC also reports grants from Singapore Ministry of Health's National Medical Research Council; research support from Singapore Institute for Clinical Sciences, Agency for Science Technology and Research, during the conduct of the study; has a patent Vitamin B12 and its use for gestational diabetes pending; has a patent Myo-inositol and one or more probiotics for use in type 2 diabetes pending; has a patent Vitamin B12 and for use in type 2 diabetes pending; and owns a patent for Myo-inositol and one or more probiotics for gestational diabetes. All other authors declare no competing interests.

\section{Data sharing}

The dataset supporting the conclusions of this article can be made available upon request from the corresponding author after approval is obtained from the GUSTO Executive Committee.

\section{Acknowledgments}

This study was supported by the Translational and Clinical Research Flagship Programme of the Singapore National Research Foundation through the Ministry of Health's National Medical Research Council (NMRC) of Singapore (NMRC/TCR/004-NUS/2008, NMRC/TCR/012NUHS/2014). Additional funding was provided by the Singapore Institute for Clinical Sciences, Agency for Science Technology and Research (A*STAR). KMG is supported by the National Institute for 
Health Research (NIHR) through the NIHR Southampton Biomedical Research Centre and by the EU Erasmus + Capacity Building Project and Seventh Framework Programme (FP7/2007-2013; EarlyNutrition and ODIN projects, grant numbers 289346 and 613977). We thank the GUSTO study group, operational managers, research fellows, study coordinators, and data management team. We greatly appreciate voluntary participation of all participants, and cooperation of KK Women's and Children's Hospital and National University Hospital.

\section{References}

1 Domingues-Montanari S. Clinical and psychological effects of excessive screen time on children. J Paediatr Child Health 2017; 53: 333-38.

2 WHO. Guidelines on physical activity, sedentary behaviour and sleep for children under 5 years of age. Geneva, Switzerland: World Health Organization, 2019.

3 Viner R, Davie M, Firth A. The health impacts of screen time: a guide for clinicians and parents. Edinburgh, Scotland: Royal College of Paediatrics and Child Health, 2019.

4 L'enfant, l'adolescent, la famille et les écrans-Appel à une vigilance raisonnée sur les technologies numériques. French Academy of Sciences, 2019. https://www.academie-sciences.fr/pdf/rapport/ appel_090419.pdf (accessed Dec 18, 2019).

5 Melkevik O, Torsheim T, Iannotti RJ, Wold B. Is spending time in screen-based sedentary behaviors associated with less physical activity: a cross national investigation. Int J Behav Nutr Phys Act 2010; 7: 46.

6 Forde C, Hussey J. How Children Use Active Videogames and the Association Between Screen Time and Physical Activity. Games Health J 2015; 4: 312-17.

7 Marshall SJ, Biddle SJH, Gorely T, Cameron N, Murdey I. Relationships between media use, body fatness and physical activity in children and youth: a meta-analysis. Int J Obes Relat Metab Disord 2004; 28: 1238-46.

8 Fakhouri TH, Hughes JP, Brody DJ, Kit BK, Ogden CL. Physical activity and screen-time viewing among elementary school-aged children in the United States from 2009 to 2010. JAMA Pediatr 2013; 167: 223-29.

9 Hale L, Guan S. Screen time and sleep among school-aged children and adolescents: a systematic literature review. Sleep Med Rev 2015; 21: $50-58$.

10 Cespedes EM, Gillman MW, Kleinman K, Rifas-Shiman SL, Redline S, Taveras EM. Television viewing, bedroom television, and sleep duration from infancy to mid-childhood. Pediatrics 2014; 133: e1163-71.

11 Radesky JS, Schumacher J, Zuckerman B. Mobile and interactive media use by young children: the good, the bad, and the unknown. Pediatrics 2015; 135: 1-3.

12 Pedišić Ž. Measurement issues and poor adjustments for physical activity and sleep undermine sedentary behaviour researchthe focus should shift to the balance between sleep, sedentary behaviour, standing and activity. Kinesiology 2014; 46: 135-46.

13 Tremblay MS, Carson V, Chaput JP, et al. Canadian our Movement Guidelines for Children and Youth: an integration of physical activity, sedentary behaviour, and sleep. Appl Physiol Nutr Metab 2016; 41 (suppl 3): S311-27.

14 Chen B, Bernard JY, Padmapriya N, et al. Socio-demographic and maternal predictors of adherence to 24-hour movement guidelines in Singaporean children. Int J Behav Nutr Phys Act 2019; 16: 70.

15 Chastin SF, Palarea-Albaladejo J, Dontje ML, Skelton DA. Combined effects of time spent in physical activity, sedentary behaviors and sleep on obesity and cardio-metabolic health markers: a novel compositional data analysis approach. PLoS One 2015; 10: e0139984.

16 Aitchison J. The statistical analysis of compositional data. J R Stat Soc B 1982; 44: 139-60.
17 Leite ML. Applying compositional data methodology to nutritiona epidemiology. Stats Methods Med Res 2016; 25: 3057-65.

18 Flood R, Bloemsma M, Weltje GJ, et al. Compositional data analysis of Holocene sediments from the West Bengal Sundarbans, India: geochemical proxies for grain-size variability in a delta environment. Appl Geochem 2016; 75: 222-35.

19 Dumuid D, Stanford TE, Martin-Fernández JA, et al. Compositiona data analysis for physical activity, sedentary time and sleep research. Stat Methods Med Res 2018; 27: 3726-38.

20 Soh SE, Tint MT, Gluckman PD, et al. Cohort profile: Growing Up in Singapore Towards healthy Outcomes (GUSTO) birth cohort study. Int J Epidemiol 2014; 43: 1401-09.

21 van Hees VT, Gorzelniak L, Dean León EC, et al. Separating movement and gravity components in an acceleration signal and implications for the assessment of human daily physical activity. PLoS One 2013; 8: e61691.

22 Sabia S, Cogranne P, van Hees VT, et al. Physical activity and adiposity markers at older ages: accelerometer vs questionnaire data. J Am Med Dir Assoc 2015; 16: 438.e7-13.

23 van Hees VT, Sabia S, Anderson KN, et al. A novel, open access method to assess sleep duration using a wrist-worn accelerometer PLoS One 2015; 10: e0142533.

24 Hildebrand M, Hansen BH, van Hees VT, Ekelund U. Evaluation of raw acceleration sedentary thresholds in children and adults. Scand J Med Sci Sports 2017; 27: 1814-23.

25 Hildebrand M, VAN Hees VT, Hansen BH, Ekelund U. Age group comparability of raw accelerometer output from wrist- and hip-worn monitors. Med Sci Sports Exerc 2014; 46: 1816-24.

26 Kingman JFC. Random discrete distributions. J R Stat Soc Series B 1975; 37: 1-15.

27 Maier MJ. DirichletReg: Dirichlet regression for compositional data in R. 2014. https://cran.r-project.org/web/packages/DirichletReg/ vignettes/DirichletReg-vig.pdf (accessed Dec 18, 2019).

28 Ferrari S, Cribari-Neto F. Beta regression for modelling rates and proportions. J Appl Stat 2004; 31: 799-815.

29 Egozcue JJ, Pawlowsky-Glahn V, Mateu-Figueras G, Barcelo-Vidal C. Isometric logratio transformations for compositional data analysis. Math Geol 2003; 35: 279-300.

30 Padmapriya N, Aris IM, Tint MT, et al. Sex-specific longitudinal associations of screen viewing time in children at 2-3 years with adiposity at 3-5 years. Int J Obes 2019; 43: 1334-43.

31 Stiglic N, Viner RM. Effects of screentime on the health and wellbeing of children and adolescents: a systematic review of reviews. BMJ Open 2019; 9: e023191.

32 DuRant RH, Baranowski T, Johnson M, Thompson WO. The relationship among television watching, physical activity, and body composition of young children. Pediatrics 1994; 94: 449-55.

33 Hardy LL, Mihrshahi S, Bellew W, Bauman A, Ding D. Children's adherence to health behavior recommendations associated with reducing risk of non-communicable disease. Prev Med Rep 2017 8: 279-85.

34 Chen B, van Dam RM, Tan CS, et al. Screen viewing behavior and sleep duration among children aged 2 and below. BMC Public Health 2019; 19: 59

35 Hale L, Kirschen GW, LeBourgeois MK, et al. Youth screen media habits and sleep: sleep-friendly screen behavior recommendations for clinicians, educators, and parents. Child Adolesc Psychiatr Clin N Am 2018; 27: 229-45.

36 Rideout V. The common sense census: Media use by kids age zero to eight. San Francisco, CA: Common Sense Media, 2017: 263-83.

37 VAN Loo CMT, Okely AD, Batterham MJ, et al. Wrist acceleration cut points for moderate-to-vigorous physical activity in youth. Med Sci Sports Exerc 2018; 50: 609-16. 